\title{
CARACTERÍSTICAS CONSTRUTIVAS E FUNCIONAIS DAS AGULHAS DE RADIOFREQUÊNCIA BIPOLARES RESFRIADAS PARA REDUZIR O SANGRAMENTO NAS RESSECÇÕES HEPÁTICAS
}

\author{
Construction of radiofrequency cold bipolar needles and its functional aspects to reduce the \\ bleeding in hepatic resections
}

José Artur SAMPAIO, Fábio Luiz WAECHTER, Thiago Luciano PASSARIN, Cristine Kist KRUSE, Mauro NECTOUX, Paulo Roberto Ott FONTES, Luiz Pereira LIMA

Trabalho realizado no Serviço de Cirurgia Hepatobiliopancreática e Transplante Hepático da Santa Casa de Porto Alegre, Porto Alegre, RS, Brasil

DESCRITORES - Radiofrequência bipolar. Hepatectomia. Sangramento.

Correspondência:

José Artur Sampaio,

e-mail: jartur@terra.com.br

Fonte de financiamento: não há

Conflito de interesses: não há

Recebido para publicação: 22/03/2011 Aceito para publicação: 19/04/2011

HEADINGS - Bipolar radiofrequency. Hepatectomy. Bleeding.
RESUMO - Racional - Para diminuir o sangramento em ressecções hepáticas diversas opções tecnológicas têm sido divulgadas, dentre elas a radiofrequência. A intenção dos vários métodos é evitar o clampeamento vascular, fazer menor dissecção hepática e obter menor sangramento. Objetivo - Apresentar uma nova técnica de agulhas paralelas de radiofrequência bipolar desenvolvidas pelos próprios autores e os detalhes técnicos de seu uso. Métodos - O sistema de agulhas apresenta dois eletrodos paralelos (18 gauge cada) de $25 \mathrm{~cm}$ de comprimento, separados entre si por uma distância de $1,5 \mathrm{~cm}$, onde apenas os $4 \mathrm{~cm}$ distais dissipam energia gerada por um eletrobisturi bipolar. Estas agulhas são refrigeradas através de um sistema de resfriamento interno por fluxo contínuo de água destilada gelada estéril a 0 oC, cuja temperatura é mantida através da presença de gelo, formado também por água destilada estéril. A operação é realizada sob anestesia geral, não sendo utilizados cateteres venosos centrais durante ou após o procedimento. As incisões realizadas podem ser subcostal direita e mediana supra-umbilical. Resultados A experiência inicial de sua utilização nas ressecções hepáticas mostraram média de 87 minutos, tamanho médio da incisão abdominal de $14 \mathrm{~cm}$ e sangramento médio de $58 \mathrm{ml}$. Nenhum paciente do grupo inicial recebeu transfusão de sangue ou derivados. Conclusão - As agulhas de radiofrequência bipolares resfriadas são viáveis e reduzem o sangramento nas ressecções hepáticas.

ABSTRACT - Background - To reduce bleeding in liver resection various technological options have been disclosed, among them the radiofrequency. The intent of the various methods is to avoid vascular clamping, less liver dissection and minimize bleeding. Aim - To present a new technique of parallel bipolar radiofrequency needles developed by the authors and the technical details. Methods - The needle system has two parallel electrodes (18 gauge each) of $25 \mathrm{~cm}$ in length, separated by a distance of $1.5 \mathrm{~cm}$, and only distal $4 \mathrm{~cm}$ dissipate energy generated by a bipolar electrocautery. These needles are cooled by an internal cooling system for continuous flow of cold sterile distilled water at $0^{\circ} \mathrm{C}$, whose temperature is maintained through the presence of sterile distilled water ice. The operation is performed under general anesthesia and is not used central venous catheters during or after the procedure. The incisions may be right subcostal and median supra-umbilical. Results - The inical use in liver resection showed an average 87 minutes operation time, average size of abdominal incision of $14 \mathrm{~cm}$ and $58 \mathrm{ml}$ of blood loss during surgery. No patient in the inical group received transfusion of blood or blood products. Conclusion - The bipolar radiofrequency cooled needles are viable and reduce bleeding in liver resection 


\section{INTRODUÇÃO}

$\mathrm{P}$ ara diminuir o sangramento em ressecções hepáticas diversas opções tecnológicas têm sido divulgadas, dentre elas a radiofreqüência ${ }^{1,3,4,5}$. A intenção dos vários métodos é evitar o clampeamento vascular, fazer menor dissecção hepática e obter menor sangramento. Os autores têm por objetivo apresentar uma nova técnica de agulhas paralelas de radiofrequência bipolar desenvolvidas por eles próprios e os detalhes técnicos de seu uso.

\section{MÉTODO}

\section{Técnica: as agulhas de radiofrequência}

O sistema de agulhas montado pelos autores apresenta dois eletrodos paralelos (18 gauge cada) de $25 \mathrm{~cm}$ de comprimento, separados entre si por uma distância de $1,5 \mathrm{~cm}$, onde apenas os $4 \mathrm{~cm}$ distais dissipam energia gerada por um eletrobisturi bipolar (Figura 1). Estas agulhas são refrigeradas através de um sistema de resfriamento interno por fluxo contínuo de água destilada gelada estéril a $0^{\circ} \mathrm{C}$, cuja temperatura é mantida através da presença de gelo, formado também por água destilada estéril.

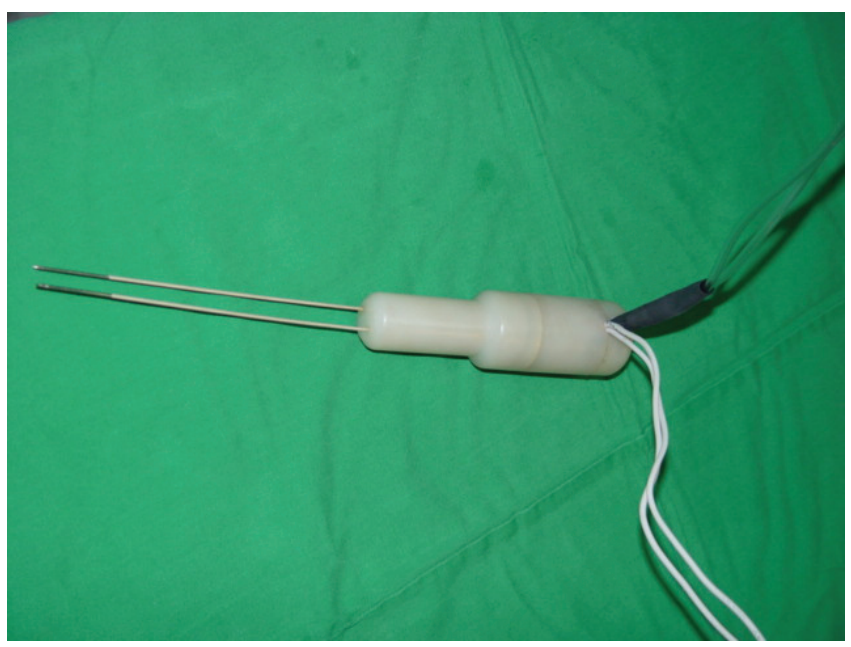

FIGURA 1 - Manopla com duas agulhas refrigeradas de radiofrequência

O eletrobisturi funciona como fonte da energia elétrica que gera a corrente elétrica necessária para a desnaturação do tecido alvo. No caso do dispositivo usado, a frequência nominal é de $500 \mathrm{kHz}$ modulada por $40 \mathrm{kHz}$. A potência disponível pelo aparelho alterna entre 4 e 80 watts. Este aparelho é dotado de controle de potência aplicada e de dispositivo de monitoramento da resistência do tecido entre os eletrodos, desligando o aparelho quando a resistência subir acima de um limite de segurança que evita a cauterização e necrose total do tecido.
O objetivo é gerar área de tecido desnaturado que propicia a formar linha de corte, através da qual uma lâmina de bisturi convencional possa cortar o parênquima sem sangramento. A área de necrose compreende $1 \mathrm{~cm}$ de largura por $1 \mathrm{~cm}$ de extensão por 4 $\mathrm{cm}$ de profundidade, deixando apenas $0,5 \mathrm{~cm}$ de tecido desnaturado junto ao parênquima remanescente, já que a secção com lâmina convencional é executada junto à borda das agulhas que estão em contato com a peça a ser removida. Esta área ocorre exatamente entre os eletrodos ativos, em volume que se estende até cerca de $80 \%$ da parte ativa exposta (Figura 2).

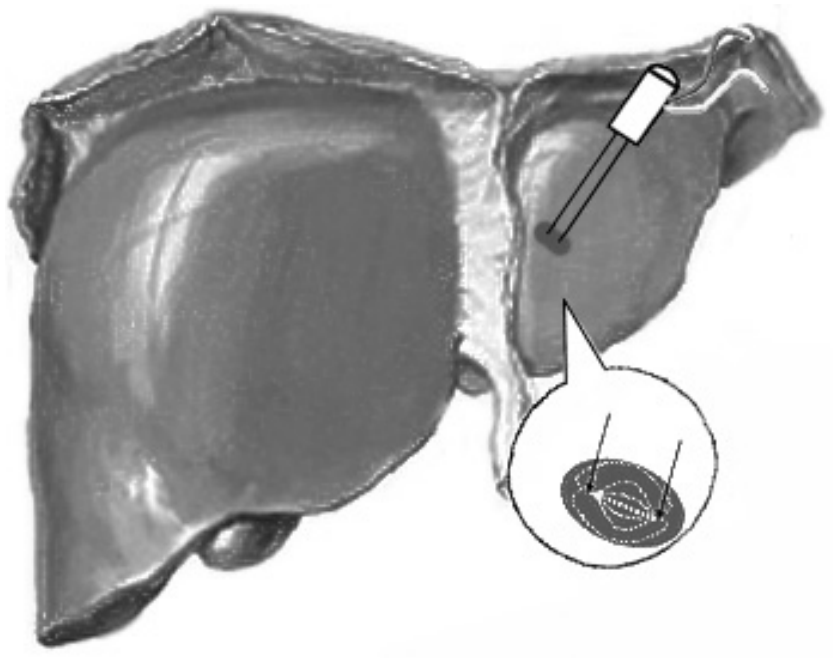

FIGURA 2 - Utilização das agulhas de radiofrequência criando área de necrose hepática para posterior secção exangue com bisturi de lâmina convencional

A tensão elétrica alternada gerada pelo eletrobisturi é aplicada pelos eletrodos, gerando gradiente de densidade de corrente elétrica na região entre os mesmos. Como o tecido da região é composto por células com determinada concentração de íons em solução aquosa, peculiar ao volume intracelular, a circulação da corrente elétrica tem baixa resistência no início do processo. Entretanto, a manutenção da circulação desta corrente alternada faz com que a resistência subitamente baixe, intensificando o calor gerado pelo efeito Joule. Este aquecimento faz com que o tecido comece a desnaturar, com consequente perda de líquido, gerando aumento gradativo da resistência do tecido entre os eletrodos. Isto ocorre até que se alcancem níveis em que há progressiva diminuição do efeito Joule. No momento em que a resistência atinja níveis de segurança pré-determinados, o eletrobisturi desliga a fonte de tensão. A temperatura gerada no tecido por este efeito chega a cerca de $80^{\circ} \mathrm{C}$. Os eletrodos são resfriados para que o tecido em contato com os mesmos não fique necrosado e aderido a eles (os quais aumentam, em muito, a resistência elétrica do tecido), garantindo assim fluxo contínuo de corrente elétrica entre os mesmos e desnaturação mais gradual 
e uniforme em todo o volume compreendido entre os eletrodos ativos.

\section{Técnica ciúrgica}

A operação é realizada sob anestesia geral, não sendo utilizados cateteres venosos centrais durante ou após o procedimento. As incisões realizadas podem ser subcostal direita e mediana supra-umbilical. Nos casos em que incisão subcostal direita é utilizada, a mesa cirúrgica deve ser lateralizada em um ângulo variável entre $15^{\circ}$ e $30^{\circ}$ para a esquerda.

Após abertura e inventário da cavidade abdominal, ecografia hepática transoperatória com transdutor em forma de $\mathrm{T}$ de $7 \mathrm{MHz}$ deve ser realizada em todos os casos para confirmação do número e posição das lesões e excluir possíveis nódulos não detectados previamente no parênquima. Necessidade de dissecção dos ligamentos do fígado é restrita aos pacientes submetidos à hepatectomias direita ou esquerda, objetivando a mobilização e exposição da área a ser removida. A demarcação da linha de ressecção com eletrocautério monopolar define a área a ser removida, permitindo que se obtenha uma distância mínima de 1 $\mathrm{cm}$ do tumor.

Sempre com controle ecográfico, a introdução das agulhas deve ser iniciada pela borda livre do fígado em direção ao interior do parênquima. Uma vez posicionada as agulhas, o processo de coagulação é iniciado, sendo interrompido quando a impedância tecidual elevada é aferida. Neste momento, o processo de coagulação tecidual é obtido entre as agulhas, assim como por uma distância de $0,5 \mathrm{~cm}$ ao redor e no eixo formado entre elas. O parênquima hepático pode então ser seccionado com bisturi de lâmina convencional exatamente no meio da linha da necrose observada, seguindo, tanto em extensão, como em profundidade, até a completa ressecção segmentar, sem necessidade de utilização de qualquer sutura ou cola biológica.

Após lavagem da cavidade, ao término da operação, dreno tubular de silicone $28 \mathrm{~F}$ pode ser colocado na dependência das condições locais antes do fechamento por planos da parede.

A experiência inicial de sua utilização nas ressecções hepáticas mostrou média de 87 minutos, tamanho médio da incisão abdominal de $14 \mathrm{~cm}$ e sangramento médio de $58 \mathrm{ml}$. Nenhum paciente do grupo inicial recebeu transfusão de sangue ou derivados (os resultados preliminares e a experiência em série de casos estão publicados no $\operatorname{ABCD} 24(2)^{2}$.

\section{CONCLUSÃO}

As agulhas de radiofrequência bipolares resfriadas são viáveis e reduzem o sangramento nas ressecções hepáticas

\section{REFERÊNCIAS}

1. Haemmerich D, Chachati L, Wright AS, Mahvi DM, Lee Jr. FT, Webster JG. Hepatic radiofrequency ablation with internally cooled probes: Effect of coolant temperature on lesion size. IEEE Trans Biomed Eng 2003; 50(4): 493-500.

2. Sampaio JA, Waechter $F L$, Passarin $T L$, Kruse $C K$, Nectoux $M$, Fontes PRO, Lima LP. É possível diminuir o sangramento em hepatectomias sem a realização de exclusão vascular total ou parcial? Resultados do uso de radiofrequência bipolar com agulhas resfriadas. ABCD Arq Bras Cir Dig. 2011; 24(2):159-163

3. Stella M, Percivale A, Pasqualini M, Profeti A, Gandolfo N, Serafini G, Pellicci R. Radiofrequency-assisted liver ressection. Gastrointest Surg 2003; 7(6): 797-801.

4. Weber JC, Navarra G, Jiao LR, Nicholls JP, Jensen SL, Habid NA. New technique for liver resection using heat coagulative necrosis. Ann Surg 2002; 236(5): 560-563.

5. Zacharoulis D, Asopa V, Navarra G, Nicholls JP, Jensen SL, Habib NA. Hepatectomy using intraoperative ultrasound-guided radiofrequency ablation. Int Surg 2003; 88(2): 80-82. 